\title{
Overcoming Application Integration Challenges Using Middleware: An Overview
}

\author{
Urang, Awajionyi Samuel ${ }^{1}$ and Ojekudo, Nathaniel Akpofure ${ }^{2}$ \\ Department of Computer Science, IgnatiusAjuru University of Education, Port Harcourt, Rivers State, Nigeria ${ }^{1,2}$
}

\begin{abstract}
No modern business operates in a vacuum. Commercial processes are constantly evolving, as is Software applications that companies rely on for critical tasks such as enterprise Resource Planning (ERP), supply chain management and financial analysis and reports. Therefore, the application integration has become increasingly important for anyone who wants to connect an older company applications to newer cloud applications, guarantee access to shared data for mobile and local devices users and navigate the digital transformation with the least possible interruption (and hopefully, great improvement over) productivity and profitability. An informed approach to integrate business applications is essential to create integration solution that leverages big data and runs all your individual applications together gently. Often, the easiest way to integrate applications is to manually program point-to-point interactions between two applications. But over time, as new integrations are added, it becomes unsustainable. Monitoring dozens or hundreds of manual integrations is very difficult. Therefore, the current best practice for integrating applications is the use of middleware to consolidate and federate the integration architecture.
\end{abstract}

Keywords: Application Integration, Middleware, Application Integration Challenges, Enterprise Application Integration

\section{INTRODUCTION}

From cutting-edge mobile applications to legacy systems, your business probably depends on a mix of individual applications that need to communicate without problems, and often not. Application integration is the process of connecting discrete commercial applications to reduce overhead, particularly in Information Technology (IT), while increasing scalability and productivity and efficiency throughout the organization.

Enterprise Application Integration (EAI) is the fusion and optimization of dataset and workflows connecting two different software applications, frequently a fresh internet application with an old local application. Application integration is usually a difficult process, especially when integrating existing applications with new applications or new web services. Given the enormous scope of this topic, I could literally write a book about successful implementation.

However, some of the basic business requirements include:

i. Adequate connectivity between platforms

ii. Business rules and logic for data transformation.

iii. Longevity of business processes.

iv. Flexible business processes

v. Flexibility in hardware, software and business objectives.

The need for application integration generally arises when a company adopts a new software application to improve or replace an obsolete business process. The new application is desirable due to its efficiency, but problems arise during implementation because the new software must interact, usually in ascending and descending processes, with legacy applications. The integration of applications consists in creating interoperability and solving the data quality problems introduced by the new applications.

Most companies use commercial applications such as Supply Chain Management (SCM), Enterprise Resource Planning (ERP) or Customer Relationship Management (CRM). When all commercial processes are filtered through these excellent applications, everything works smoothly. But these applications do not always offer the most innovative solution for a common business problem. Cloud and mobile applications often give employees an easier way to do certain aspects of their work, and when that happens, the organization must create integrations between the old and the new.

Today, Information Technology (IT) has turn out to be crucial in all organizations, playing role ranging on or after support and support the enterprise to support its growth. Large multinational companies operating in global markets, the incessant growth of computer technology, at the same time as continuously extensive, isolated and interconnected corporate information systems basic change of the economy [1]. These transformations and trends as Globalization, mergers and acquisitions and the growing proliferation of trade have reformed the Competition game in the market. 
In such a competitive and constantly evolving market, technological and commercial standards are rarely generally accepted, sometimes it is sometimes better to develop a competitive standard than to join an existing standard. In addition, the different components of the information system were generally considered as independent systems in their own right before the idea of their integration was inserted. This leads to a situation in which, most of the time, there are no standards. It is therefore essential that user organizations seek the integration of software systems that have been built without or with limited common interaction assumptions. [2] In addition, to survive and thrive in these competitive markets, companies must find a way to cope with rapid changes in internal and external environments. In order to respond quickly to In an evolving environment, business functions must be efficiently integrated into a single system. Use information technology and share data with third parties in the market. [3]

To define the domain of these systems, a variety of terms such as Business Application Integration (EAI), Application Integration (AI), System Integration (SI), Value Chain Integration (VCI), supply chain integration (SCI), Extended Business Integration (EBI) and integration presented in the literature [4]. In the context of this report, the term Commercial Integration (EI) is adopted to designate the field of integration and employment insurance systems are used to designate the associated information systems.

There have been several attempts to define business integration. According to Linthicum [5] the definition as unlimited exchange of data and business processes between all connected applications and data though this explanation illustrates the extent of the region, it provides limited information. Summary of the diverse size of the region, limiting its perception from the technical point of analysis.

Lee et al. in [3] it provides a more complete definition of employment insurance as an IT business term for schemes, methods and tools aimed at modernizing, consolidating and coordinating all IT functionalities in a business. This meaning gives a more complete analysis of the area, always limiting the business Integration with the internal functionalities of a company.

Themistocleous and Irani provided a taxonomy for the domain of EI in [6], dividing it into intra-organizational, hybrid and inter-organizational subcategories. The first subcategory includes the integration of intra-organizational systems, such as packaged and customized systems. The second describes the integration of business with consumer applications. The applications in this subcategory are characterized as hybrids, because in some cases these applications function as intra-organizational $\mathrm{AI}$ and in others as inter-organizational applications. The last subcategory includes the integration of $\mathrm{B} 2 \mathrm{~B}$ (Business to Business) applications and is classified according to the degree (loose, tight) of integration.

\section{RELATED WORK}

The main forces are asking for a change in organizational life given the technology-organization relationship. These include the migration of organizations to a "greater complexity" with a "global presence", "severe economic pressures", "the desire within companies to strengthen innovation and be more entrepreneurial", and "the incorporation of social values to more participatory actions, focused on learning and various management practices "[7].

Given these strengths, studies in organizational sciences generally coincide in the evolution of organizational forms towards decentralized and more flexible approaches and structures. In this evolution, electronic communication technologies make it possible to change organizational forms by offering capabilities to overcome the limitations of time and distance and the main obstacles. [7]

Given the inter-organizational characteristic inherent in market transactions, there has been a growing interest in interorganizational forms. From the perspective of inter-organizational forms, electronic communication plays an important catalytic role. By allowing the transition to electronically assisted relationships with other companies, the integration of the companies results in new forms of coupling between organizations. [7] Various scientific publications have addressed the issue of the advantages and effects of business integration in companies from different points of view.

Before the systems of business integration, the integration of different systems required rewriting the codes in the origin and destination systems, which consumed a lot of time and money. [3] However, unlike traditional integration, IE uses special middleware that acts as a bridge between the various integration applications [3]. In this way, all applications can freely communicate with each other through a common interface layer, rather than through point-topoint integration [3]. This could lead to a significant reduction in the number of connections between information systems in a company, given the worst case scenario, a state of spaghetti systems with connections [1] (see Figure 1, A and B).

Service-oriented architecture (SOA) is an emerging paradigm of software architecture. The OASIS group has defined service-oriented architecture as "a paradigm for organizing and using distributed capabilities that may be under the control of different property domains" [8]. In the context of SOA, services are defined as "a mechanism to access one or more capabilities, where access is provided using a prescribed interface and is exercised in accordance with the restrictions and policies specified by the service description" [8 ]. The application of SOA in the field of EI seems to offer promising advantages, mainly due to the flexibility of this architecture, which leads to greater business agility. 
Vol. 9, Issue 2, February 2020

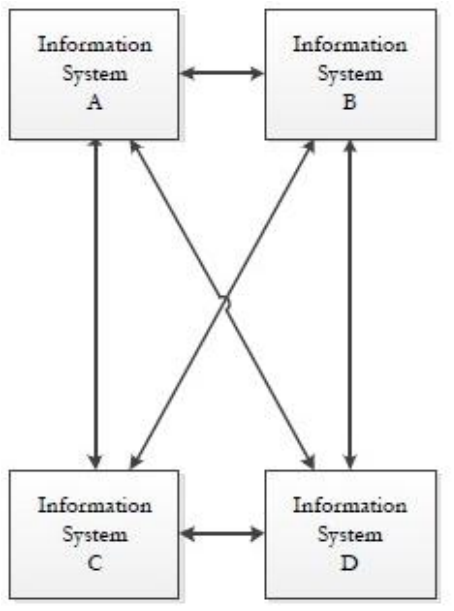

(A)

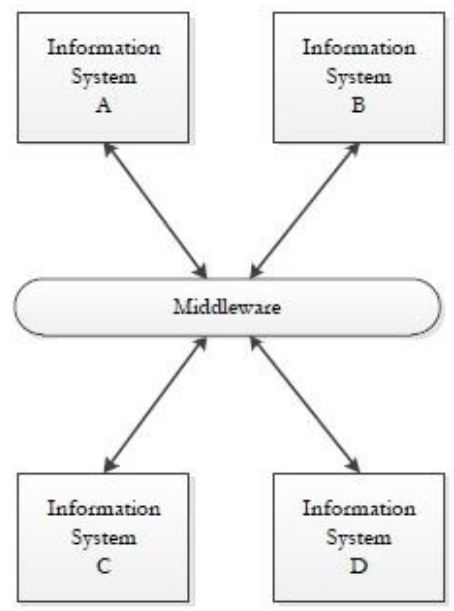

(B)

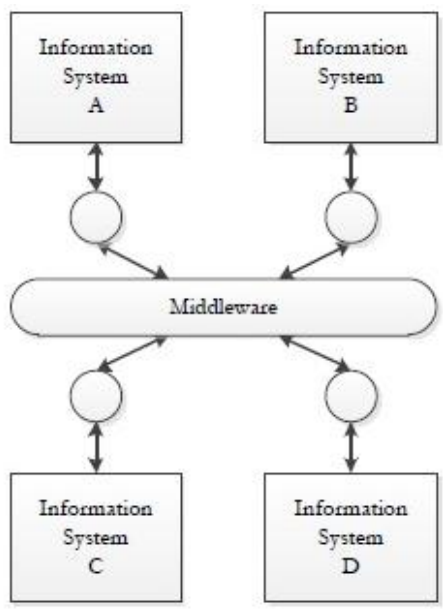

(C)

Fig. 1 Traditional Integration (A); EI (B); EI with SOA approach(C)(Source: J. Lee et al, 2003)

The authors of [9] propose a framework as a tool for decision-making regarding the adoption of employment insurance. The proposed framework suggests criteria classified into four integration layers, namely the connectivity layer, the transport layer, the translation layer and the process automation layer.

The benefits of EI can be classified into five different types; organizational (for example, more organized business processes), management (for example, ROI), strategic (for example, increasing collaboration between partners), technical (for example, obtaining data, integration of objects and processes) and operational (for example, reduce costs) [10]. However, Swatman et al. discuss that in practice it is difficult to distinguish between technical aspects of integration and organizational issues of implementation and integration [11].

While it suggests flexibility or agility, such as the ability to respond quickly to new business opportunities, such as the final objectives of EI, the Business Integration Council offers cycle time reductions, cost reductions and cost control as system benefits of business integration [3]. Welcoming these benefits, Chari and Seshadri argue that "the adoption of standards-based integration solutions is the most promising way to reduce long-term integration costs and facilitate a flexible infrastructure" [11].

Themistocleous and Irani [6] consider the advantages of IE as a reduction in integration time, more flexible and maintainable solutions and the facilitation of migration to new technologies due to compliance with EI systems with common standards [6]. They argue that these benefits ultimately translate into reduced overall integration costs due to the reduction in integration time and maintenance costs.

Ruh et al. Take on the challenge of determining the effects of employment insurance from an organizational point of view. They claim that an integrated infrastructure allows companies to improve their performance, increase their productivity and increase the quality of the services offered to their customers [6]. Similarly, Themistocleous and Irani claim that EI is strengthening supply chains [6]. Manouvrier and Menard [1] also emphasize that improving service quality is the most significant advantage of integration systems.

From another point of view, Shin [12] states that IE is generally effective for large companies, larger than a critical point. They point out that to rationalize the initial fixed cost of EI systems, companies must have conditions such as a mass of complex information systems.

In addition to increasing responsiveness and adaptive capacity and the ability to manage external exchanges optimally, Manouvrier and Menard [1] suggest accelerating time to market as another effect of EI.

Addressing the problem of determining the effects of EI, Themistocleous and Irani in [13] and [14] suggests that information systems that benefit from integration with others can probably be considered $\mathrm{n}$ 'without further beginning or definitive end. The authors affirm that these systems are evolutionary entities that develop and develop over time, according to the business environment.

Analyzing the benefits of business data integration, the authors of [15] suggest business objectives such as improved productivity, improved data accuracy, greater agility and flexibility, and system replacement / organizational mergers.

Middleware

An intermediate layer (as shown in Figure 2) provides generic interfaces through which integrated systems can communicate. Middleware performs tasks such as routing and data transmission. Each of the interfaces defines a business process provided by an application1. Adding and replacing applications will not affect another application. 
Vol. 9, Issue 2, February 2020

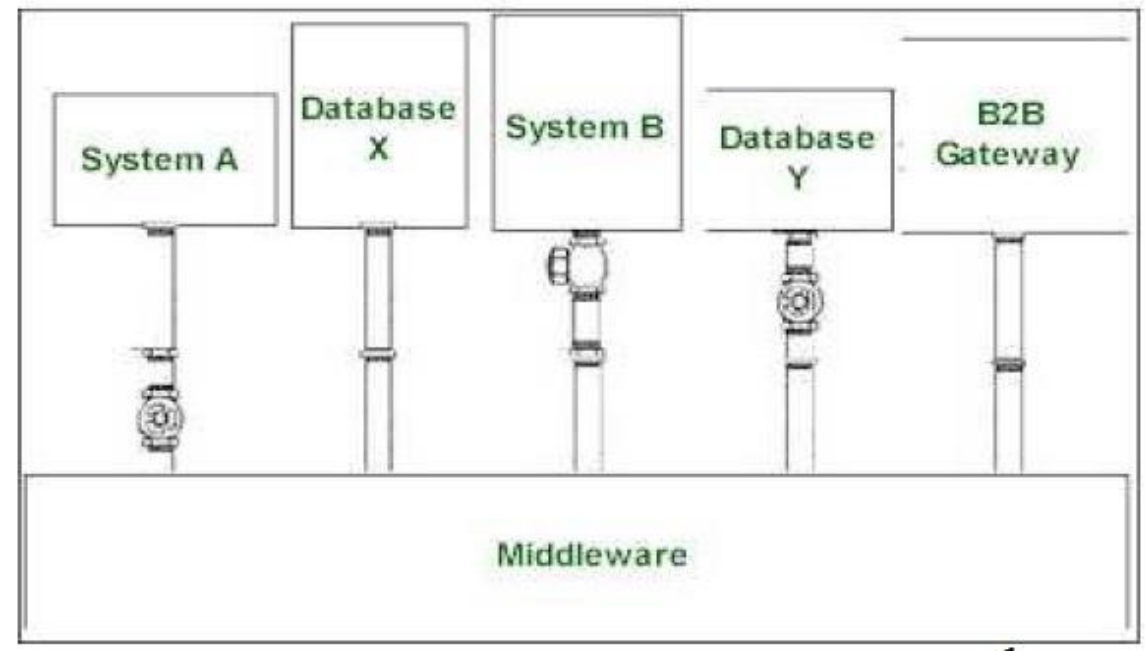

Fig. 2 Middleware Based Integration (Source: Florence Lin, 2005)

Compared to the point-to-point approach, middleware-based integration can easily support a greater number of applications and does not require much maintenance. Despite these advantages, it should be borne in mind that there is additional initial complexity in the configuration of the middleware and the conversion of existing applications to use the middleware APIs.

After selecting the integration architecture, a decision must be made regarding the integration method. Organizations need to appreciate both business processes and data. Then they must select those that require integration. This can take several dimensions; In EAI, there are four common types of integration:

- Data level integration

- Application level integration

- Method level integration

- User interface (UI) - level integration

\section{Data-level}

At this level, the back-end data stores are integrated to allow data movement between them. In a nutshell, the information can be extracted from a database, processed as necessary, and then updated in another database. In an EAI business, this could mean extracting data from hundreds of databases and thousands of tables. For this reason, the preservation of the integrated application data remains a problem. For example, a table may have dependencies on others, and the integrated application may be the sole executor of these dependencies.

Data level integration can be based on push or pull. Transmission-based integration occurs when an application performs SQL queries in the database of another application; the data is sent to the database of another application. In contrast, extraction-based integration is used when an application requires passive notification of changes in the data of another application1.

The economic benefits of data integration give you an advantage over other approaches. In fact, in general, the application does not change; the code is not modified and, therefore, the costs of modifying, testing and implementing the application are not incurred2.

Data level integration should be used when the application being integrated does not provide any API or client interface. This is usually represented as the only option with custom applications without an application API.

\section{Application-level}

This refers to the use of interfaces contained in custom or packaged applications such as SAP, Peoplesoft or Baan. These interfaces are used to provide access to business processes and information. This approach is probably the best way to integrate applications because it allows you to call business logic to preserve data integrity. Developers can group many applications so that information and business logic can be shared. This approach is used more widely and is preferred because it is transparent to the integrated application and the integrity of the application data is preserved.

\section{Method-level}

In fact, it is a more complicated and less used form of application level integration. Common operations in multiple applications are combined into a single front-end application. For example, you can access the method to update a customer record from many applications without having to rewrite each method in the respective application. Because all applications that interact with integrated applications do so through this front-end application, method-level integration requires that integrated applications support RPC (remote procedure call) or distributed component 
technology. The disadvantage is that changing the built-in application API will break the components of the front-end application and the applications that depend on them. Under these conditions, it is generally more appropriate to opt for application level integration using middleware.

\section{User interface (UI)-level integration}

Although more primitive, this approach is necessary and useful. Applications can be grouped together and use their user interfaces as a common point of integration: this approach is known as user interface integration at the proxy level. There is a second type based on scripts. There are those who consider this approach to be unstable and, although not preferable, integration at the user interface level should be used in cases where the database cannot be easily or directly accessed. data, or when your business logic is integrated into the user interface. For example, mainframes that do not provide data stores or public APIs. Many client / server applications integrate business logic into the client. Accessing and maintaining data integrity in such cases can only be achieved through integration into the user interface.

\section{CONCLUSION}

Application Integration (AI) or Enterprise Application Integration (EAI) is a well-established research field, which provides methodologies, techniques and tools to design and implement integration solutions. Companies rely on EAI to reuse the applications that are available within their software ecosystems to support their business processes. There are currently several open-source integration platforms available for companies to assist the design and implementation of their integration solutions.

Applications use middleware for a variety of reasons. The middleware can be used to "soften" the appearance of the application and hide its distributed nature, eliminating any difference in terms of operating systems, hardware or protocols. The use of middleware also makes it easier for developers to have a uniform interface to work, so that the different components of the application can be reused and ported to other locations.

In recent years, the industry has undergone a shift towards open source middleware as more mature and stable solutions have emerged. Open source middleware has a lot to recommend: users are free to modify the source code for their own needs and objectives, and you have complete visibility and transparency in the solution to know that your applications are secure. Here are three of the most popular options for enterprise middleware: Red Hat JBoss EAP, IBM WebSphere and Oracle WebLogic.

These three middleware solutions are solid options, so the choice ultimately depends on your situation. If you trust other products in the IBM or Oracle ecosystems, for example, it also makes sense to use your middleware offerings. In the end, it is worth talking to sales representatives about the particular situation of your organization, so you can be better informed and make the right decision for your business.

The features work for middleware will be based on comparing the middleware software from the open-source middleware integration application, to know the best, using the advantages and disadvantages including its success factor in Enterprise Application Integration (EAI).

\section{REFERENCES}

[1]. B. Manouvrier and L. Ménard, Application Integration: EAI, B2B, BPM and SOA. Wiley-IEEE Press, 2008 , p. 256.

[2]. A.-W. Scheer and F. Habermann, "Making ERP a success," Communications of the ACM, vol. 43, no. 4, pp. 57-61, 2000.

[3]. J. Lee, K. Siau, and S. Hong, "Enterprise integration with ERP and EAI," Commun. ACM, vol. 46, no. 2, pp. 54-60, Feb. 2003.

[4]. M. Themistocleous and Z. Irani, "Novel taxonomy for application integration," Benchmarking: An International Journal, vol. 9, no. 2, pp. 154$165,2002$.

[5]. D. S. Linthicum, Enterprise application integration. Addison-Wesley Longman Ltd. Essex, UK, UK, 2000 , p. 400.

[6]. M. Themistocleous and Z. Irani, "Benchmarking the benefits and barriers of application integration," Benchmarking: An International Journal, vol. 8, no. 4, pp. 317-331, 2001.

[7]. J.Fulk and G.DeSanctis, "Electronic communication and changing organizational forms," Organization Science, vol. 6, no.4, pp.337-349, 1995.

[8]. C. MacKenzie, K. Laskey, F. McCabe, P. Brown, and R. Metz, "Reference model for service oriented architecture 1.0," Architecture, no. October. OASIS open, 2006.

[9]. M. Themistocleous and Z. Irani, "Towards a novel framework for the assessment of enterprise application integration packages," in Proceedings of the 36th Annual Hawaii International Conference on System Sciences, 2003.

[10]. M. Themistocleous, "Justifying the decisions for EAI implementations: a validated proposition of influential factors," Journal of Enterprise Information Management, vol. 17, no. 2, pp. 85-104, 2004.

[11]. E. Folmer and J. Verhoosel, "State of the Art on Semantic IS Standardization, Interoperability \& Quality," UT, CTIT, TNO en NOiV, 2011.

[12]. I.Shin, “Adoption of Enterprise Application Software \& Firm Performance," Small Business Economics, vol. 26, no. 3, pp. $241-256$, Apr. 2006.

[13]. T. Puschmann and R. Alt, Enterprise application integration-the case of the Robert Bosch Group, vol. 00, no. c. IEEE Comput. Soc, 2001 , p. 10.

[14]. M. Themistocleous, Z. Irani, K. Psannis, and A. Vrehopoulos, “Application Integration of Information Technology: Classification of Benefits and Barriers," Information Systems, pp. 156- 164, 2001.

[15]. R. Gleghorn, "Enterprise application integration: a manager"s perspective," IT professional, no. December, pp. 17-23, 2005

[16]. F., Lin, (2005) Enterprise application integration (EAI) techniques, 3C05-CW2 Florence Lin March 2005 